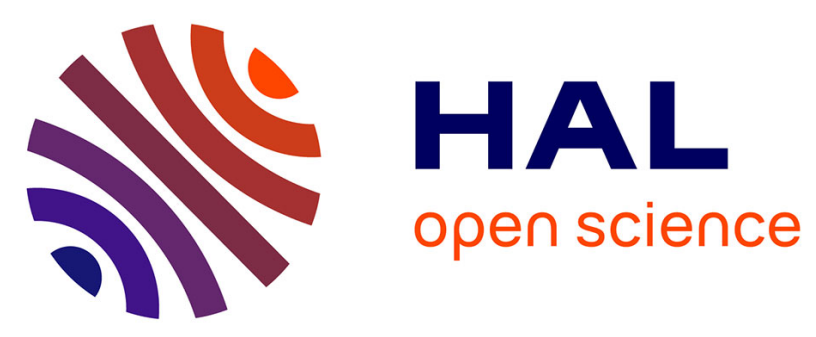

\title{
An unusual mechanism of building up of high magnetization blocking barrier in an octahedral alkoxide Dy 3+ -based Single-Molecule Magnet
}

Jérôme Long, Aleksei O Tolpygin, Ekaterina Mamontova, Konstantin A Lyssenko, Munirah D Albaqami, Dan Liu, Liviu F Chibotaru, Yannick Guari, Joulia Larionova, Alexander A Trifonov

\section{To cite this version:}

Jérôme Long, Aleksei O Tolpygin, Ekaterina Mamontova, Konstantin A Lyssenko, Munirah D Albaqami, et al.. An unusual mechanism of building up of high magnetization blocking barrier in an octahedral alkoxide Dy 3+ -based Single-Molecule Magnet. Inorganic Chemistry Frontiers, 2021, 8 (5), pp.1166-1174. 10.1039/D0QI01267J . hal-03164904

\author{
HAL Id: hal-03164904 \\ https://hal.science/hal-03164904
}

Submitted on 10 Mar 2021

HAL is a multi-disciplinary open access archive for the deposit and dissemination of scientific research documents, whether they are published or not. The documents may come from teaching and research institutions in France or abroad, or from public or private research centers.
L'archive ouverte pluridisciplinaire HAL, est destinée au dépôt et à la diffusion de documents scientifiques de niveau recherche, publiés ou non, émanant des établissements d'enseignement et de recherche français ou étrangers, des laboratoires publics ou privés. 


\title{
An unusual mechanism of building up of high magnetization blocking barrier in an octahedral alkoxide $\mathrm{Dy}^{3+}$-based Single- Molecule Magnet
}

\author{
Jérôme Long, ${ }^{\text {a }}$ Aleksei O. Tolpygin, ${ }^{b, c}$ Ekaterina Mamontova, ${ }^{a}$ Konstantin A. Lyssenko, ${ }^{c, d}$ Munirah \\ D. Albaqami, ${ }^{\mathrm{e}}$ Dan Liu, ${ }^{\mathrm{f}}$ Liviu F. Chibotaru, ${ }^{* \mathrm{~g}}$ Yannick Guari, ${ }^{\mathrm{a}}$ Joulia Larionova, ${ }^{\text {a }}$ and Alexander A. \\ Trifonov* ${ }^{\mathrm{b}, \mathrm{c}}$

\begin{abstract}
We report a new octahedral $\left[\mathrm{Dy}\left(\mathrm{OCPh}_{3}\right)_{2}(\mathrm{THF})_{4}\right]\left[\mathrm{BPh}_{4}\right]$ luminescent SMM exhibiting a massive crystal-field splitting and an anisotropic barrier of $1385 \mathrm{~cm}^{-1}$. Magnetic measurements combined with ab initio analysis reveal a novel mechanism behind the high blocking barrier based on the quench of one-phonon transitions between the three low-lying crystal-field multiplets due to large energy gaps between them exceeding the available phonon energies and forcing the activated relaxation to proceed through the fourth doublet. The observed nonetheless short relaxation time is due to appreciable non-axial anisotropy, which opens a tunnelling relaxation path via interaction with the nuclear spins. Reducing the equatorial crystal field quenches drastically the quantum tunnelling of magnetization, allowing for full exploitation of the high blocking barrier of the complex as in the best known SMMs.
\end{abstract}

\section{Introduction}

Lanthanide-based materials continue to fascinate the scientific community owing to their exceptional optical and magnetic properties. ${ }^{1}$ In particular, the ability of lanthanide coordination complexes to exhibit slow relaxation of magnetization, often associated with a magnetic bistability of molecular origin called Single-Molecule Magnet (SMM) behavior, opens stimulating perspectives in data storage, spintronics or quantum computing. ${ }^{2-5}$ However, one of the major drawbacks associated to SMMs concerns their relatively moderate "working" temperature which, in the wide majority of the investigated systems, necessitates liquid helium technology. The specific chemical design of the coordination environment around the lanthanide ion could however be used to address these issues. The key quantity underlying the magnetization blocking is the anisotropy (magnetization blocking) barrier opposing the reversal of the magnetic moment aligned to the anisotropy axis of the complex. The efficiency of SMMs could be at first sight related to the height of the anisotropy barrier, $\Delta$, and to the magnetization blocking temperature $T_{\mathrm{B}}{ }^{6}$ The blocking of the magnetization is a manifestation of the axial magnetic anisotropy of the complex, which arises at its turn from the interplay of strong spin orbit-coupling with preponderant axial crystal-field (CF) on the metal site. Compared to transition metal ions, lanthanides exhibit large spin-orbit coupling and unquenched orbital momentum resulting in the presence of an axial $\mathrm{CF}$, in a large splitting of atomic $J$ multiplet into doublet states. A net advantage of lanthanides is a large value of their total angular momentum $J$ resulting in low-lying doublets of strong axiality (the highest of which defining $\Delta$ ) even in the absence of pronounced axial CF. ${ }^{7}$ The CF splitting of these doublets may be boosted by the choice of specific coordination environment controlled by the ligands which impacts the geometry/symmetry of the complexes. ${ }^{8}$ To this purpose, an efficient recently described strategy to engineer SMMs with optimized magnetic relaxation coming from a thermally activated process consists in designing linear mononuclear complexes by locating the electronic density of oblate ions, such as $\mathrm{Dy}^{3+}$, in an axial CF generated by two close ligands along an axis. ${ }^{9-10}$ In this sense, the $\mathrm{Dy}^{3+}$-ligand distances in the axial direction should be as short as possible, while those from the equatorial plane as long as possible or either inexistent. ${ }^{10}$ However, this situation is frequently compromised by the presence of additional underbarrier relaxation processes of different physical origins, such as the Quantum Tunneling of the Magnetization (QTM) providing a direct transition between the ground state doublet as well as spin-lattice Raman and direct relaxations involving intricate mechanisms that decrease the observed energy barrier. $^{3,6,10-16}$

Following the prediction of multi-thousand-kelvin barriers for low-coordinated Ln(III) complexes, ${ }^{7}$ major breakthroughs have been achieved within the last few years with the report of high energy barrier SMMs. ${ }^{10}, 14,17-22$ In general, this necessitates obtaining a strong axiality not only for the ground state but also for excited states, which is particularly difficult to achieve and rationalize from a synthetic point of view. To the best of our knowledge, two families of mononuclear $\mathrm{Dy}^{3+}$ based SMMs exhibiting high energy barriers, which follow the above mentioned strategy, have recently been reported. The first one belongs to organometallic complexes involving cyclopentadienyl $\left(\mathrm{Cp}^{\mathrm{R}}\right)$ derivatives, such as dysprosium metallocenes. ${ }^{14,}$ 23-24 For instance, the heteroleptic complex $\left[\mathrm{Dy}\left(\mathrm{Cp}^{\mathrm{iPr} 5}\right)\left(\mathrm{Cp} \mathrm{p}^{*}\right)\right]\left[\mathrm{B}\left(\mathrm{C}_{6} \mathrm{~F}_{5}\right)_{4}\right]$ shows an enhanced anisotropy due to the synergistic combination of large $\mathrm{Cp}$-Dy-Cp angles with short Dy-Cp distances leading to the highest energy barrier of $1541 \mathrm{~cm}^{-1}$ and hysteresis observed up to $80 \mathrm{~K}^{23}$ The second family consists in a series of coordination complexes of pentagonal bipyramidal geometry $\left[\operatorname{DyXX}\left(\mathrm{L}_{e q}\right)_{5}\right]^{+}$, where $\mathrm{X}$ and $X^{\prime}$ ligands are alkoxide, aryloxides, siloxides, halides located in the axial position and $\mathrm{L}_{\mathrm{eq}}$ are solvent molecules (pyridine or THF) in the equatorial plane. ${ }^{19,25-27}$ The highest energy barrier of $1262 \mathrm{~cm}^{-1}$ in this family is obtained for the $\left[\mathrm{Dy}\left(\mathrm{O}^{\mathrm{t}} \mathrm{Bu}\right)_{2}(\mathrm{py})_{5}\right]\left[\mathrm{BPh}_{4}\right]$ compound. ${ }^{19}$ An existence of a linear correlation has been demonstrated between the $\mathrm{Dy}-\mathrm{X}\left(\mathrm{X}^{\prime}\right)$ bond lengths in the axial position (which vary between 2.023 and 
$2.829 \AA$ ) and the value of the energy barrier, which increases as the bond lengths decreases. However, the question of the $\mathrm{O}-\mathrm{Dy}-\mathrm{O}$ axial angle linearity has not been clarified and it is noteworthy that its perfect linearity has not been obtained for this family. It has been assumed that the magnetization blocking arises from the high axiality of low-lying Kramers (KD) doublets. Moreover, the luminescence surprisingly has never been investigated for these complexes despite the great interest in the synthesis of multifunctional SMMs and the possibility of experimental correlation between both properties.

We report in this article the synthesis, experimental and theoretical investigations of a luminescent $\mathrm{Dy}^{3+}$-based SMM exhibiting an unusual octahedral geometry and a perfectly linear O-Dy-O angle in the axial direction inducing a huge CF splitting. This conducts to the appearance of a very high magnetization blocking barrier via an unprecedented mechanism based on the suppression of all one-phonon transitions involving the three low-lying KDs forcing in turn the relaxation through the fourth excited state. Remarkably, the height of the anisotropic barrier of $1385 \mathrm{~cm}^{-1}$ is not related to a strong axiality of the complex but rather to a particular situation arising from a lack of suitable high energy phonons with respect to the CF splitting, distinguishing this complex from the family of previously published compounds with pentagonal bipyramidal geometry.

\section{Experimental}

\section{General Conditions}

All experiments were performed in evacuated tubes by standard Schlenk and glove-box techniques with the rigorous exclusion of traces of moisture and air. After drying over $\mathrm{KOH}$, THF was purified by distillation from sodium/benzophenone ketyl. $\mathrm{Dy}\left(\mathrm{CH}_{2} \mathrm{SiMe}_{3}\right)_{3}(\mathrm{THF})_{2}{ }^{28}$ was prepared according to literature procedure. $\mathrm{Ph}_{3} \mathrm{COH}$ and $\left[\mathrm{Et}_{3} \mathrm{NH}\right]\left[\mathrm{BPh}_{4}\right]$ were purchased from Alfa Aesar. IR spectra were recorded as Nujol mulls on $\mathrm{KBr}$ plates on a Bruker-Vertex 70 spectrophotometer. The N, C, H elemental analyses were carried out in the microanalytical laboratory of the IOMC by means of a Carlo Erba Model 1106 elemental analyser with an accepted tolerance of 0.4 unit on carbon $(\mathrm{C})$, hydrogen $(\mathrm{H})$, and nitrogen (N). Lanthanide metal analysis was carried out by complexonometric titration. ${ }^{29}$

\section{Synthesis of $\left[\mathrm{Dy}\left(\mathrm{OCPh}_{3}\right)_{2}(\mathrm{THF})_{4}\right]\left[\mathrm{BPh}_{4}\right](1)$}

A solution of $\mathrm{Ph}_{3} \mathrm{COH}(0.73 \mathrm{~g}, 2.82 \mathrm{mmol})$ in $20 \mathrm{~mL}$ of THF was added to a solution of $\mathrm{Dy}\left(\mathrm{CH}_{2} \mathrm{SiMe}_{3}\right)_{3}(\mathrm{THF})_{2}(0.80 \mathrm{~g}, 1.41$ $\mathrm{mmol})$ in THF $(20 \mathrm{~mL})$. The reaction mixture was stirred at room temperature for $10 \mathrm{~min}$. and the solution of $\left[\mathrm{Et}_{3} \mathrm{NH}\right]\left[\mathrm{BPh}_{4}\right](0.59 \mathrm{~g}, 1.41 \mathrm{mmol})$ in THF $(20 \mathrm{~mL})$ was added. The reaction mixture was stirred overnight at ambient temperature. Slow concentration of the resulting solution at room temperature afforded 1 as colourless crystals in $78 \%$ yield (1.41 g). $\mathrm{C}_{78} \mathrm{H}_{82} \mathrm{BDyO}_{6}$ (1288.79): calc. C 72.69, $\mathrm{H} 6.41$, Dy 12.61; found C 72.33, H 6.09, Dy 12.42 .

\section{X-Ray crystallography}

Crystals of $1\left[\mathrm{C}_{78} \mathrm{H}_{82} \mathrm{BDyO}_{6}, \mathrm{FW}=1288.74\right]$ are monoclinic, space group $C 2 / c$, at $120(2) \mathrm{K} a=25.613(3), b=13.7071(11), c$ $=17.8695(14) \AA, \beta=92.698(3)^{\circ}, V=6266.6(9) \AA^{3}, Z\left(Z^{\prime}\right)=4(0.5)$, $\left.\mathrm{d}_{\text {calc }}=1.366 \mathrm{gcm}^{-3}, \mu\left(\mathrm{Mo}_{\mathrm{K} \alpha}\right)\right)=12.48 \mathrm{~cm}^{-1}$. Intensities of 38310 reflections were measured with Bruker APEX-II CCD $\left[\lambda\left(\mathrm{Mo}_{\mathrm{K \alpha}}\right)=\right.$ $0.71072 \AA$, $2 \theta<66^{\circ}$ and 11640 independent reflections $\left(R_{\text {int }}=\right.$ 0.0282 ) were used in the further refinement. The structure was solved by direct method and refined by the full-matrix least-squares technique against $F^{2}$ in the anisotropic-isotropic approximation. Analysis of the Fourier density synthesis as well as displacement parameters clearly show that one of the THF molecules is disordered by two positions with occupancies $0.45: 0.55$. The refinement of disordered part has been performed with restraints (DFIX and EADP). Hydrogen atoms in 1 were placed in calculated positions and were refined in the "riding" model with $U(H)_{\text {iso }}=1.2 U_{\text {eq }}$ of their parent atoms The refinement converged to $\mathrm{WR}_{2}=0.0576$ and $\mathrm{GOF}=1.036$ for all independent reflections $\left(R_{1}=0.0256\right.$ was calculated against $F$ for 9438 observed reflections with $1>2 \sigma(I))$. The maximum and minimum values of difference density were 0.524 and $-0.604 \mathrm{e}$ $\AA^{-3}$. All calculations were performed using SHELXTL-2017. Crystallographic data for the structure 1 was deposited at the Cambridge Crystallographic Data Centre as supplementary no. CCDC 1943271.

\section{Magnetic Measurements}

Magnetic susceptibility data were collected with a Quantum Design MPMS-XL SQUID magnetometer working in the range 1.8-350 $\mathrm{K}$ with the magnetic field up to 7 Tesla. The sample was prepared in a glove box. The data were corrected for the sample holder and the diamagnetic contributions calculated from the Pascal's constants. The AC magnetic susceptibility measurements were carried out in the presence of a 3 Oe oscillating field in zero or applied external DC field.

\section{Photoluminescence measurements}

The solid sample was sealed in a quartz tube in an argon atmosphere (glove box). The emission and excitation spectra were recorded at $77 \mathrm{~K}$ and $295 \mathrm{~K}$ using a spectrofluorimeter Edinburgh FLS-920. The excitation source was a $450 \mathrm{~W}$ Xe arc lamp. The emission spectra were corrected for detection and optical spectral response of the spectrofluorimeter. Low temperature measurements $(77 \mathrm{~K})$ were performed using a liquid nitrogen dewar (quartz).

\section{Computational details}

All calculations were carried out with MOLCAS 8.3 package po $^{30}$ and are of CASSCF/RASSI/SINGLE_ANISO type. The active space in the CASSCF calculations included 9 electrons in 7 orbitals for Dy ( $4 f$ orbitals of $\mathrm{Dy}^{3+}$ ion). Because of a large number of atoms in 1 , CASPT2 calculations could only be done for a fragment of the structure (see the Supporting Information for the details). On their basis, CASPT2 corrections to the CF of the complex where calculated as described elsewhere. ${ }^{31}$ The resulting spin- 
orbital multiplets where used for the calculation of magnetic properties ( $g$-tensors, magnetic axes, local magnetic susceptibility, magnetization blocking barrier etc.) using the SINGLE_ANISO module. ${ }^{30,32}$

\section{Results and Discussions}

\section{Synthesis and structure}

Our synthetic strategy to obtain linear dysprosium cationic alkoxide complex relies on the one pot alkane elimination reaction of $\left[\mathrm{Dy}\left(\mathrm{CH}_{2} \mathrm{SiMe}_{3}\right)_{3}(\mathrm{THF})_{2}\right]$ with a two-fold molar amount of $\mathrm{Ph}_{3} \mathrm{COH}$ and one equivalent of $\left[\mathrm{Et}_{3} \mathrm{NH}\right]\left[\mathrm{BPh}_{4}\right]$ in THF solution (Fig. 1a). Slow concentration of the reaction mixture at ambient temperature affords the cationic complex $\left[\mathrm{Dy}\left(\mathrm{OCPh}_{3}\right)_{2}(\mathrm{THF})_{4}\right]\left[\mathrm{BPh}_{4}\right](\mathbf{1})$ as colorless crystals in $78 \%$ yield. $\mathrm{X}$-Ray diffraction on single crystals indicates that $\mathbf{1}$ crystallizes in the monoclinic $\mathrm{C} 2 / \mathrm{c}$ space group with a unique crystallographic complex in the unit cell. The structure could be described as a separated ion pair containing a cationic dysprosium moiety [Dy $\left.\left(\mathrm{OCPh}_{3}\right)_{2}(\mathrm{THF})_{4}\right]^{+}$(Fig. 1, Table S1 ESI), the electroneutrality being ensured by a tetraphenylborate anion. Two alkoxides and four THF ligands constitute the coordination sphere of the $\mathrm{Dy}^{3+}$ giving a coordination number of six. lies on a symmetry element and the asymmetric unit is composed of half of the complex, resulting in the presence of only three different Dy-O distances.

\section{a}

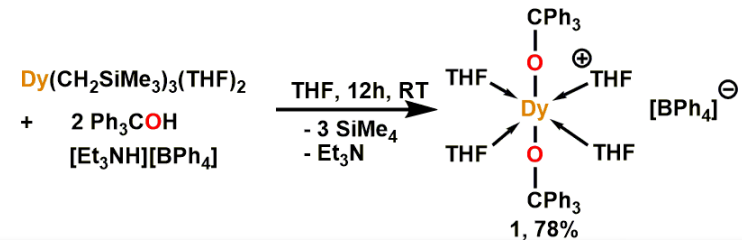

b

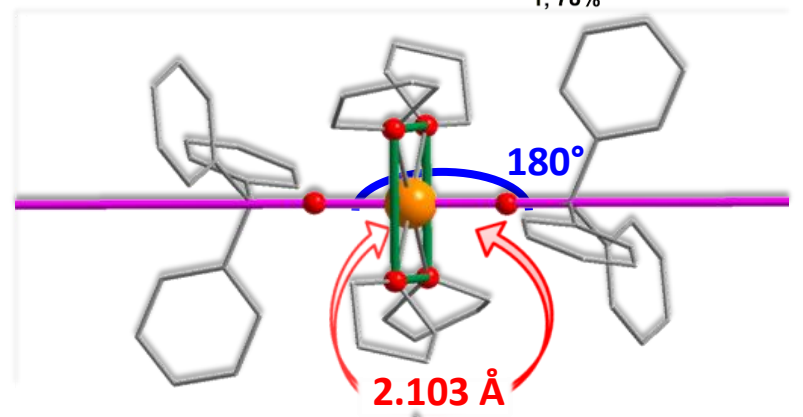

C

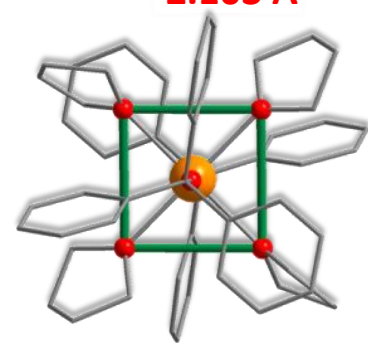

Fig. 1. a) Scheme for the synthesis of 1 . b) Molecular structure of the cationic complex 1 . Color code: orange, Dy; blue, $\mathrm{N}$; red, O; grey, C. Hydrogen atoms and anisotropic axis abtained by ab initio calculations while the green bonds represent the basal octahedral plane. c) View of 1 showing the octahedral arrangement.
Hence the two shortest $\mathrm{Dy}-\mathrm{OCPh}_{3}$ distances are equal to 2.103(1) $\AA$, while the Dy-O(THF) ones are significantly longer and equal to $2.370(1)$ and $2.398(1) \AA$. This six-coordinated geometry is a consequence of the presence of bulky $\mathrm{Ph}_{3} \mathrm{CO}^{-}$ ligands that restrain the coordination of equatorial ligands with respect to pentagonal bipyramidal complexes with less steric hindrance. ${ }^{19,25-27}$ Remarkably, the $\mathrm{Dy}^{3+}$ ion lies on a symmetry element and the asymmetric unit is composed of half of the complex, resulting in the presence of only three different Dy-O distances. Hence the two shortest $\mathrm{Dy}-\mathrm{OCPh}_{3}$ distances are equal to 2.103(1) $\AA$, while the Dy-O(THF) ones are significantly longer and equal to $2.370(1)$ and 2.398(1) $\AA$. This results in a compressed octahedral geometry which is further confirmed by the quantitative analysis using the SHAPE software (Table S2, ESI). ${ }^{33}$ Due to the $C_{i}$ symmetry of the dysprosium site, the $\mathrm{Ph}_{3} \mathrm{CO}-\mathrm{Dy}$-OCPh angle is perfectly linear $\left(180^{\circ}\right)$.The analysis of anisotropic displacement parameters in 1 clearly shows that the imposed symmetry does not lead to disorder of the Dy and oxygen atoms (Fig. S1, ESI). In comparison with other pentagonal bipyramidal alkoxide-based SMMs $\left[\mathrm{Dy}(\mathrm{OR})_{2}\left(\mathrm{~L}_{\mathrm{eq}}\right)_{5}\right]^{+},{ }^{19,26}$ the octahedral complex 1 exhibits one of the shortest Dy-O distances in the axial position with a perfectly linear angle (Table S3, ESI). Although direct correlation between complexes of different coordination number is difficult it may be tempting to compare $\mathbf{1}$ with the $\left[\mathrm{Dy}(\mathrm{OPh})_{2}(\mathrm{THF})_{5}\right]^{+}$complex exhibiting similar equatorial ligands. The presence of the phenyl groups in $\mathbf{1}$ induces a decrease in the coordination number from 7 to 6 which is usually characterized by a shortening of the Dy-ligand bond lengths. ${ }^{34}$ Owing to the presence of large tetraphenylborate anions, the shortest intermolecular $\mathrm{Dy}^{3+}-\mathrm{Dy}^{3+}$ distance of $11.261 \AA$ is relatively large indicating that the complexes are well isolated (Fig. S1, ESI).

\section{Magnetic Properties}

The magnetic properties of $\mathbf{1}$ were investigated in both, static and dynamic modes. At room temperature, the $\chi T$ value of $13.54 \mathrm{~cm}^{3} \cdot \mathrm{K} \cdot \mathrm{mol}^{-1}$ is slightly lower than the value of 14.17 $\mathrm{cm}^{3} . \mathrm{K} \mathrm{mol}^{-1}$ expected for a single $\mathrm{Dy}^{3+}$ ion (Fig. S2, ESI), suggesting a significant CF splitting. ${ }^{35}$ Upon cooling, a gentle decrease of $\chi T$ is firstly observed. However, a sudden decrease below $10 \mathrm{~K}$ could be noticed to reach the value of 2.66 $\mathrm{cm}^{3} . \mathrm{K}_{\mathrm{mol}} \mathrm{m}^{-1}$ at $1.8 \mathrm{~K}$, reinforcing the large CF splitting assumption. The field dependence of the magnetization at 1.8 $\mathrm{K}$ shows an inflection point at low field before reaching the value of $4.90 N \beta$ under a $70 \mathrm{kOe}$ field (Fig. S2, ESI) without a clear saturation. This value is close to the theoretical one of $5.00 N \beta$ expected for a pure Ising system $\left(g_{\text {eff }, z}=20\right)$. The sigmoidal shape of the magnetization curve has been imputed to the pronounced high field-dependence of the magnetic relaxation rates. ${ }^{26}$

Investigation of the occurrence of a slow relaxation of the magnetization was performed using alternate currents (ac) measurements. Under a zero-dc field, the frequency dependence of the out-of-phase susceptibilities reveals a series of single frequency dependent peaks (Fig. 2a,b) which, remarkably, could be still observed up to $120 \mathrm{~K}$ (Fig. S3, ESI). 
The Cole-Cole plots reveal well-defined semi-circles (Fig. S4, ESI), that could be fitted with a generalized Debye model confirming a narrow distribution of relaxation times with $\alpha$ values lower than 0.3 (Table S4, ESI).

Aiming at investigating the relaxation dynamics of the magnetization, the temperature dependence of the relaxation time, $\tau$, was analyzed. A clear thermally activated process could be observed in the linear region of the $\ln \tau$ vs. $T^{-1}$ plot (Fig. 2c) giving an Arrhenius fit with $\Delta=1220 \pm 50 \mathrm{~cm}^{-1}$. Decreasing the temperature results in a deviation from linearity reflecting the occurrence of additional relaxation processes. The whole temperature dependence of the relaxation time could be modeled with the following equation, $\tau^{-1}=\tau_{0}^{-1} \exp (-\Delta / k T)+C T^{n}+\tau^{-1}$ QTM (Eq. 1). ${ }^{36}$ The first term accounts for a thermally activated process, while the second and third ones stand for two-phonon Raman and QTM, respectively. To avoid over-parameterization, the $n$ coefficient was successfully fixed to integer numbers (1 to 12). It was however not possible to achieve pertinent fitting by letting the QTM rate $\tau^{-1}$ QTM free. Consequently, it was fixed to the value observed at the lowest temperature, since the relaxation time becomes temperature independent. The best fitting correlation coefficient was obtained with $n=2.5$, suggesting the presence of acoustic phonons. ${ }^{37}$ The other parameters could be found in Table 1 and indicate that the relaxation involves these three processes. Zero-field slow relaxation in six-coordinate lanthanide complexes have been previously reported with lower barriers. ${ }^{38-40}$ However, the value of the anisotropy barrier of $1385 \pm 28 \mathrm{~cm}^{-1}$ is even higher than those observed in the family of pentagonal bipyramidal complex, where the highest barrier has been reported for $\left[\mathrm{Dy}\left(\mathrm{O}^{\mathrm{tBu}}\right)_{2}(\mathrm{py})_{5}\right]\left[\mathrm{BPh}_{4}\right]\left(\right.$ i.e. $\left.1260 \mathrm{~cm}^{-1}\right) \cdot{ }^{19,26}$

From these results, it appears that the QTM process could be still observed despite the optimized symmetry of the $\mathrm{Dy}^{3+}$ ion. In order to shortcut the QTM contribution, the field dependence of the relaxation time was investigated at $40 \mathrm{~K}$ (Fig. S5). It could be modeled using the equation $\tau^{-1}=D H^{4} T+$ $B_{1} /\left(1+B_{2} H^{2}\right)+K$ (Eq. 2), for which the first term accounts for the direct process (for Kramers-ion), the second one for the QTM, while the $K$ constant considers the Raman and thermally activated processes (Fig. S6, ESI). The best fit parameters could be found in Table S5 (ESI).
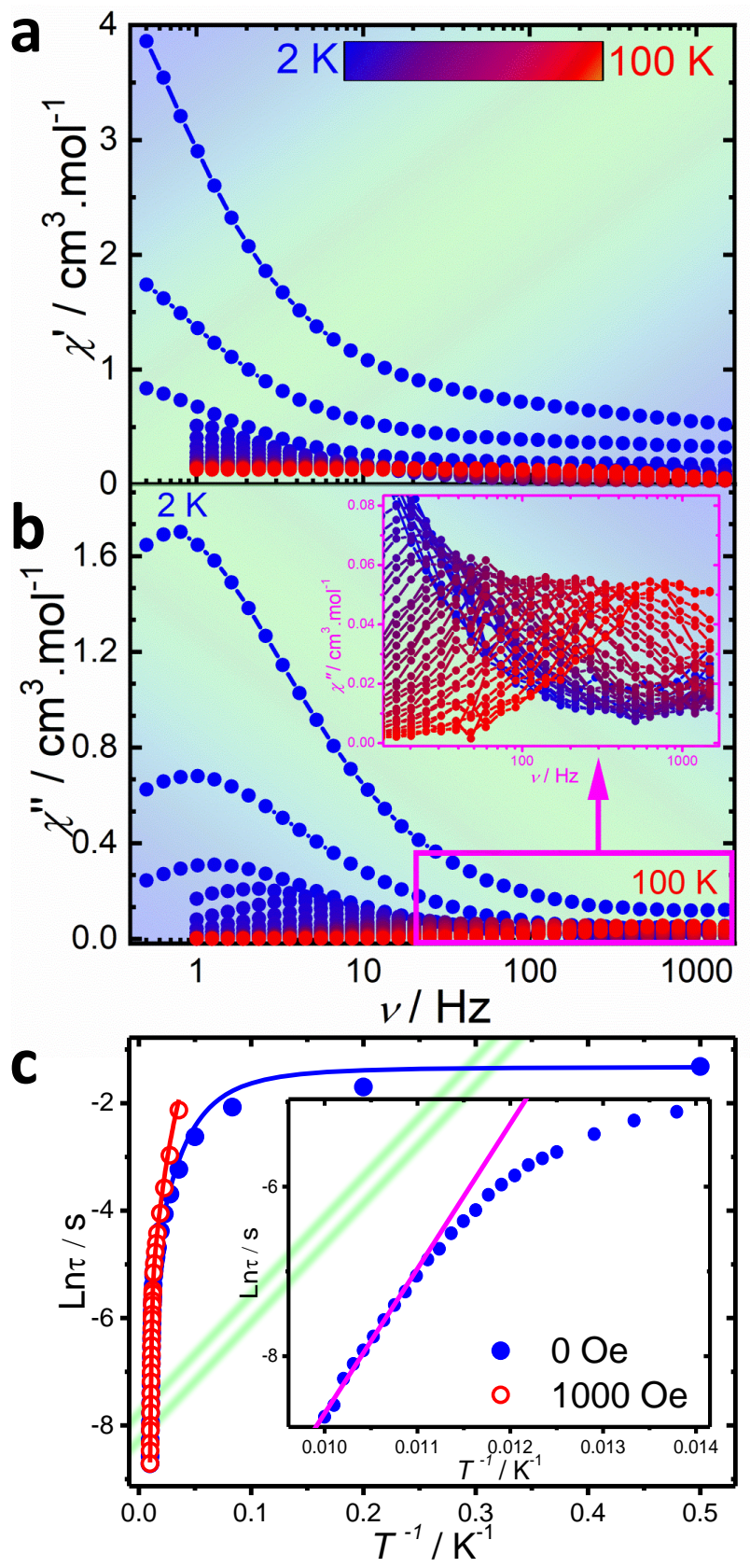

Fig. 2. a) Frequency dependence of the in-phase $\left(\chi^{\prime}\right)$ and b) out-of-phase susceptibility ( $\left.\chi^{\prime \prime}\right)$ for $\mathbf{1}$ under a zero dc field. c) Temperature dependence of the relaxation time using the ac susceptibility data for 1 . The solid lines represent the activated region showing the linear Arrhenius fit (magenta line)

The optimum field that corresponds to the highest relaxation time is found at 1000 Oe. The best fit parameters could be found in Table S5 (ESI). The optimum field that corresponds to the highest relaxation time is found at 1000 Oe. The ac susceptibility measurements performed under this optimum dc field corroborate the shortcutting of the QTM at low temperature (Fig. S7-S8, ESI), while the Cole-Cole plots (Fig. S9, ESI) could be still fitted with a generalized Debye Model (Table S6, ESI). The temperature dependence of $\tau$ can be fitted with $\tau$ ${ }^{-1}=\tau_{0}^{-1} \exp (-\Delta / k T)+C T^{n}$ (Eq. 3) (Fig. 2c, Table 1) considering that the direct relaxation process, is inoperative for such weak 
dc magnetic field. ${ }^{36}$ As expected for a field-independent thermally activated process, the anisotropic barrier is found comparable (within the uncertainty) with respect to the zerofield data.

Finally, the ability of $\mathbf{1}$ to retain its magnetization over a pertinent timescale was evaluated. Occurrence of a magnetic irreversibility could be evidenced on the zero-field cooled (ZFC)/field cooled (FC) curves at 2000 Oe (Fig. S10, ESI). Hence, a maximum of the ZFC curve corresponding to the approximate blocking temperature, $T_{\mathrm{B}}{ }^{41}$ is located at $7 \mathrm{~K}$, while a divergence of the ZFC/FC associated with a crossing of the two curves could be observed at $10 \mathrm{~K}$. This latter in lanthanide SMM was previously interpreted as an out-ofequilibrium state due to the occurrence of competitive relaxation paths. ${ }^{14,}{ }^{19-20},{ }^{42-43}$ Note that the blocking temperature value is however lower in comparison to that determined from the ZFC curve of $\left[\mathrm{Dy}\left(\mathrm{O}^{\mathrm{t}} \mathrm{Bu}\right)_{2}(\mathrm{py})_{5}\right]\left[\mathrm{BPh}_{4}\right](14$ $\mathrm{K})$, while the anisotropy energy barrier for $\mathbf{1}$ is greater. ${ }^{19}$ Yet, comparison of $T_{\mathrm{B}}$ using this method is however difficult due to the extreme temperature sweep-rate sensitivity. In our experimental conditions, a clear opening of the hysteresis loops is observed at $1.8 \mathrm{~K}$ for $\mathbf{1}$ with a significant coercive field of 280 Oe (sweeping rate $=19$ Oe. $\mathrm{s}^{-1}$ ) while it disappears at 10 K (Fig. S11, ESI).

Table 1: Fit parameters of the temperature dependence of the relaxation time for 1 .

\begin{tabular}{|c|c|c|c|c|c|}
\hline Compound & $\begin{array}{c}\Delta \\
\left(\mathrm{cm}^{-1}\right)\end{array}$ & $\tau_{0}(\mathrm{~s})$ & $n^{*}$ & $C\left(\mathrm{~s}^{-1} \cdot \mathrm{K}^{-n}\right)$ & $\tau_{\text {QTM }}(\mathrm{ms})$ \\
\hline $\mathbf{1}(0 \mathrm{Oe})$ & $\begin{array}{c}1385 \pm \\
28\end{array}$ & $\begin{array}{c}(4 \pm 1) \times \\
10^{-13}\end{array}$ & 2.5 & $\begin{array}{c}0.0042 \pm \\
0.0003\end{array}$ & 0.27 \\
\hline $\mathbf{1}(1000 \mathrm{Oe})$ & $\begin{array}{c}1338 \pm \\
30\end{array}$ & $\begin{array}{c}(8 \pm 3) \times \\
10^{-13}\end{array}$ & 3.2 & $\begin{array}{c}0.00016 \pm \\
00001\end{array}$ & - \\
\hline
\end{tabular}

*fixed parameter

\section{Luminescence}

The presence of alkoxide $\mathrm{Ph}_{3} \mathrm{CO}^{-}$ligands, which might be used as antennas for $\mathrm{Dy}^{3+}$-based emission, opens the possibility to provide luminescence properties to yield bifunctional SMMs. ${ }^{44}$

${ }^{46}$ To the best of our knowledge and at the exception of the pentagonal bipyramidal complex [Dy $\left.\left(\mathrm{CyPh}{ }_{2} \mathrm{PO}\right)_{2}\left(\mathrm{H}_{2} \mathrm{O}\right)_{5}\right] \mathrm{Br}_{3}$ $\left(\mathrm{CyPh}{ }_{2} \mathrm{PO}=\mathrm{cyclohexyl}(\right.$ diphenyl $)$ phosphine oxide $)$ with a barrier of $353 \mathrm{~cm}^{-1}$, 31 the optical properties have never been reported for the family of high energy barriers $\left[\mathrm{DyX}_{2}\left(\mathrm{~L}_{\mathrm{eq}}\right)_{5}\right]^{+}$ SMMs ( $X=$ alkoxide, aryloxides, siloxides). ${ }^{19,}$ 26-27 At room temperature and when excited at $387 \mathrm{~nm}, 1$ exhibits a broad emission band located at $475 \mathrm{~nm}$ and emission lines associated to the intra-4f $f^{9}$ transitions in the $560-600 \mathrm{~nm}$ and $650-670 \mathrm{~nm}$ regions (Fig. 3). The former large emission band most likely originates from an organic-based emission (ligands $\mathrm{Ph}_{3} \mathrm{CO}^{-}$or tetraphenylborate moieties). The excitation spectrum monitored at $590 \mathrm{~nm}$ reveals the presence of sharp lines pointing out a lanthanide direct sensitization (Fig. S12, ESI). Unfortunately, the ${ }^{4} \mathrm{~F}_{9 / 2} \rightarrow{ }^{6} \mathrm{H}_{15 / 2}$ emission transitions involving the magnetic $\mathrm{Dy}^{3+}$ ground-state, usually found around $475 \mathrm{~nm}$, overlap with the large emission band even at low temperature (Fig. S13, ESI) that precludes a detailed analysis of the CF splitting by luminescence spectroscopy. Noticeably, the most emissive transition is found at $588 \mathrm{~nm}$, which is located at slightly higher wavelength with respect to the value of $575 \mathrm{~nm}$ usually observed for dysprosium-based compounds and related to the ${ }^{4} \mathrm{~F}_{9 / 2} \rightarrow{ }^{6} \mathrm{H}_{13 / 2}$ transitions. ${ }^{44-47}$ It is well known that the optical properties of lanthanide ions are weakly affected by the coordination environment resulting in a calibrated emission wavelength range. Thus, the observed emission shift may reflect the large CF splitting of the $D y^{3+}$ even for the ${ }^{6} \mathrm{H}_{13 / 2}$ state. This represents also an appealing approach to finely tune and control the emission wavelengths. Moreover and to our knowledge, $\mathbf{1}$ exhibits one of the highest anisotropic barriers reported for a luminescent SMM.

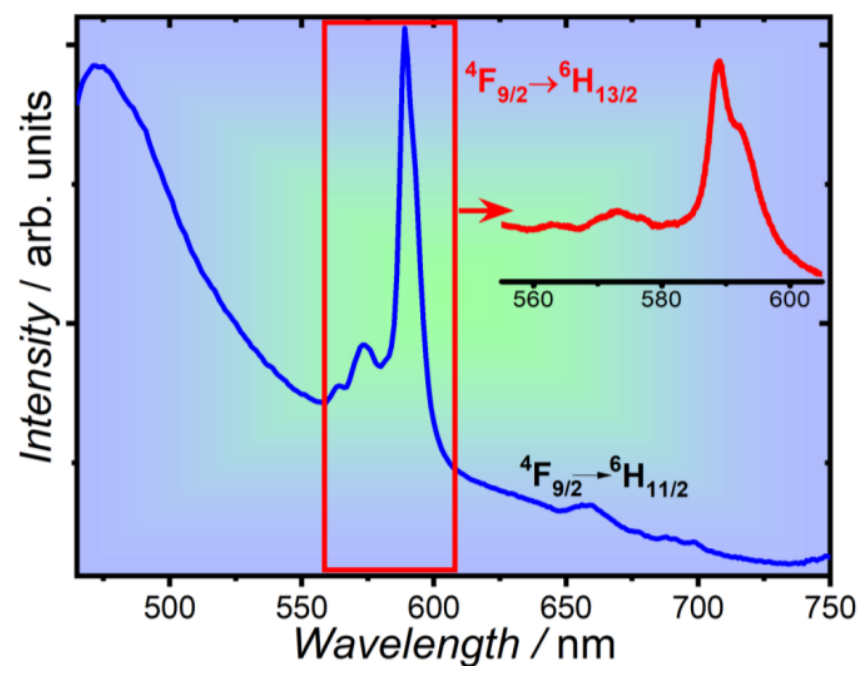

Fig. 2. Room temperature solid-state emission spectra of $\mathbf{1}$ acquired at with an excitation wavelength of $387 \mathrm{~nm}_{6}$ The intra $4 \mathrm{f}^{9} \mathrm{Dy}^{3+}$ transitions are reported. Inset: magnification of the ${ }^{4} \mathrm{~F}_{9 / 2} \rightarrow{ }^{6} \mathrm{H}_{13 / 2}$ transitions.

\section{Ab initio calculations and relaxation mechanism}

To get insight into the multiplet structure and anisotropic magnetic properties of $\mathbf{1}, a b$ initio calculations of the CASPT2/RASSI level have been undertaken as discussed elsewhere (see the Methods for the details and Computational details, ESI). ${ }^{32}$ The resulting energies and the $g$ tensor values for the eight Kramers doublets (KDs) corresponding to the ground multiplet ${ }^{6} \mathrm{H}_{15 / 2}$ of $\mathrm{Dy}^{3+}$ are shown in Tables $\mathrm{S} 12-\mathrm{S} 13$ (ESI). One can see that the overall CF splitting is $c a .1700 \mathrm{~cm}^{-1}$, i.e. twice larger than for typical Dy(III) complexes, which is undoubtedly due to a short Dy-O distance for axially coordinated $\mathrm{Ph}_{3} \mathrm{CO}^{-}$(Fig. 1), resulting in enhanced covalent contributions to $\mathrm{CF}^{48}$ Moreover, it is also found larger in comparison with the family of pentagonal bipyramidal SMMs. For instance, the $1^{\text {st }}$ excited $K D$ is expected at $725 \mathrm{~cm}^{-1}$ in $\mathbf{1}$, while it is reported at $564 \mathrm{~cm}^{-1}$ in $\left[\mathrm{Dy}\left(\mathrm{O}^{\mathrm{tBu}}\right)_{2}(\mathrm{py})_{5}\right]\left[\mathrm{BPh}_{4}\right](\mathrm{Fig}$. $\mathrm{S} 14, \mathrm{ESI}) .^{19}$ Remarkably, such large splitting is also observed for the three first KDs (Table S13, ESI). As a consequence of the short $\mathrm{Dy}-\mathrm{O}\left(\mathrm{CPh}_{3}\right)$ distances, the THF ligands will have a weaker effect due to longer Dy-O bonds, nevertheless, their contribution to CF is far from being negligible, which is manifested in a turn of anisotropy axis from the axial (Fig. 1) to a perpendicular direction starting from the third excited KD (Fig. 4). On the contrary, in complexes with strongly 
predominant axial $\mathrm{CF}$, all $\mathrm{KD}$ s should have their anisotropy axis aligned to the axial direction. 7 , 9-10, 14, 17, 19, 21, 23-24, 26, 42, 49 Note that the tetragonal CF from the four THF ligands, when superimposed on the trigonal symmetry of the two $\mathrm{Ph}_{3} \mathrm{CO}^{-}$ ligands, leads to lack of symmetry. This can be inferred from the composition of the wave functions of the eight KDs, showing a significant departure from pure $J m$ state ( $m$ is the projection of $J$ to the axial direction, Fig. 1 ) in all KDs except the ground one (Table S11, ESI). As a result, the matrix elements of transition magnetic moment (Fig. 4a), the square of which defines the electronic factor in the transition rate between the corresponding KDs components, ${ }^{7}$ are largely greater compared to strongly blocking Dy(III) SMMs (Fig. S14, ESI). ${ }^{10}$ In particular, this implies that starting with some temperatures; one should observe an activated relaxation with the blocking barrier corresponding to the energy of the first excited doublet $( \pm 2)$, i.e. $700 \mathrm{~cm}^{-1}$. This is however not the case since the extracted temperature dependence of the relaxation time (Fig. $2 \mathrm{c}$ ) is consistent with the blocking barrier of $1385 \mathrm{~cm}^{-1}$. This value does not match also the energy of the calculated second excited KD $( \pm 3)$ but agrees well with the position of the third excited one $( \pm 4)$.

Given the large energy separation between the three low-lying KDs (ca $700 \mathrm{~cm}^{-1}$ between 1 and 2, and $c a 500 \mathrm{~cm}^{-1}$ between 2 and 3) we may conclude that all one-phonon transitions involving these KDs are suppressed (Fig. 4b). This refers not only to direct and Orbach processes between these states (e.g. $-1 \rightarrow 2 \rightarrow 1,-2 \rightarrow 3 \rightarrow 2$, etc...) but also to incoherent QTM $-1 \rightarrow 1$ and $-2 \rightarrow 2$ induced by electron-phonon escape processes whose rates are determined by one-phonon transitions shown by the corresponding dashed vertical lines in Fig. 4b. In such situation, the only relaxation processes allowed in the low- $T$ domain (when the Boltzmann population of the second excited KD $( \pm 3)$ is negligible) are the QTM induced by nuclear spins and the Raman process. This agrees with the above simulations of the experimental relaxation time. Note that unlike one-phonon transitions, the multiphonon ones are well allowed for some of these KDs. Actually, given the smallness of the magnetic matrix elements, the multiphonon transitions are expected to be negligible between KD components with opposite signs of magnetization. However, they will be relevant for neighbor magnetic states (such as, e.g., -2 and -3 ), for which magnetic matrix elements are $2 \div 4$ orders of magnitude larger, and are therefore responsible for the Boltzmann population of the levels.

At $T>60 \mathrm{~K}$, when the second excited KD $( \pm 3)$ starts to be thermally populated, four new relaxation processes between the states -3 and 3 become operative, all involving the third excited KD $(n=4)$ (cf Fig. 4b): (1) the Orbach process $-3 \rightarrow-4 \rightarrow 3$; (2) the Orbach process $-3 \rightarrow 4 \rightarrow 3$; (3) the incoherent QTM $-3 \rightarrow 3$; (4) the incoherent QTM $-4 \rightarrow 4$. Remarkably, all these processes have an activation temperature dependence corresponding to the energy of the third excited KD $(n=4)$. Indeed, taking into account that the rate of transition from an excited state $n$ is scaled by the corresponding Boltzmann factor $p_{n}=\exp \left(-E_{n} / k T\right) / Z^{50}$ and that the down electron-phonon transitions (bold dashed lines in
Fig. 4b) are practically $T$-independent, ${ }^{51}$ we can write for the rates of the four relaxation processes: $\Gamma^{(1)}=\Gamma^{(2)} \approx c_{1} p_{3} \exp \left[-\left(E_{4}-\right.\right.$ $\left.\left.\left.E_{3}\right)\right] / k T\right), \Gamma^{(3)} \approx c_{3} p_{-3} \exp \left[-\left(E_{-4}-E_{-3}\right) / k T\right]$ and $\Gamma^{(4)} \approx c_{4} p_{-4}$, where $c_{n}$ are temperature-independent constants.
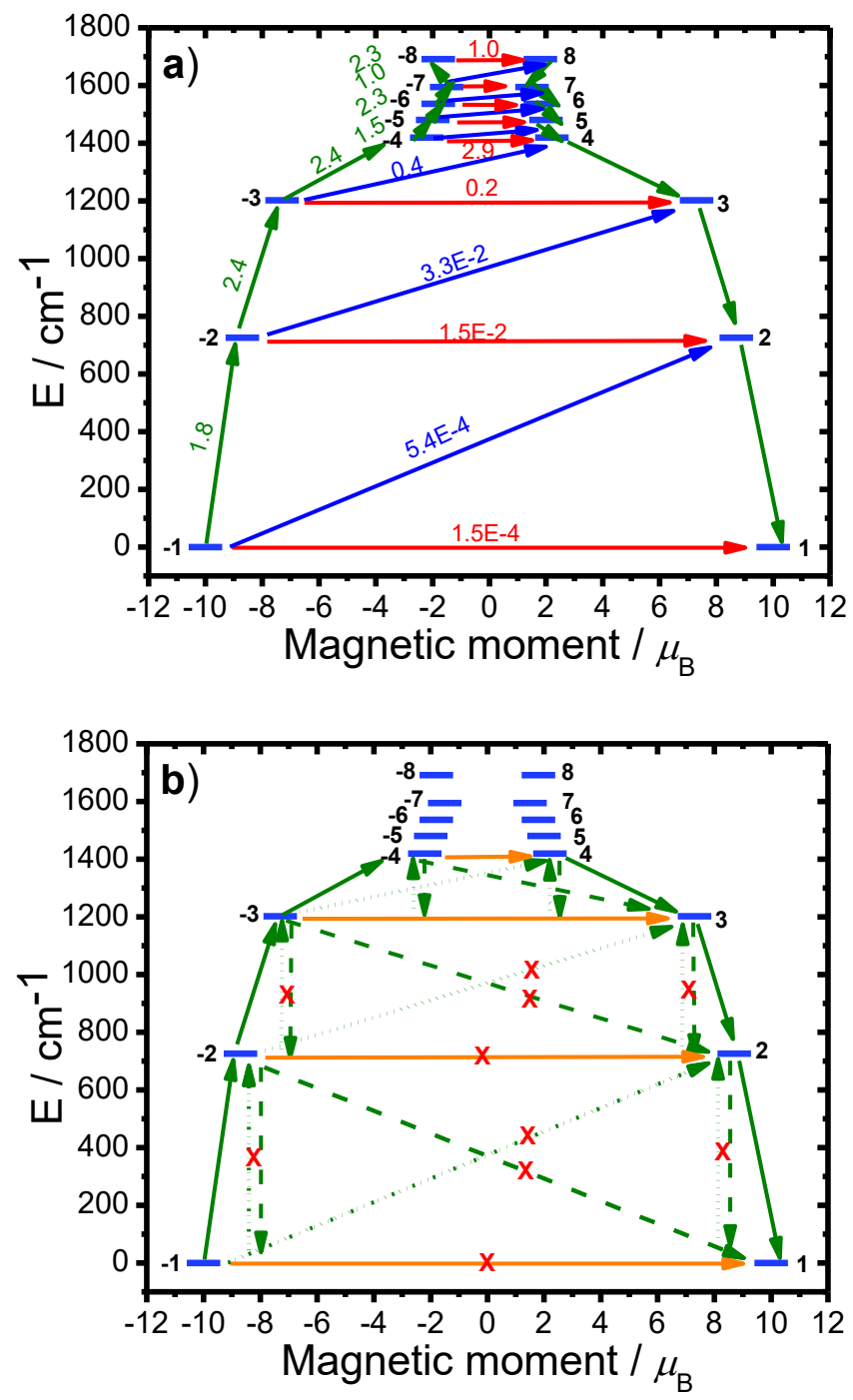

Fig. 4. Energy diagram for low-lying KDs and factors influencing the magnetic relaxation in 1 . For each KD $(n)$, the corresponding states $(-n, n)$ are placed according to their magnetic moments. The horizontal arrows show the QTM transitions within each doublet, whereas the non-horizontal arrows are spin-
phonon transition paths. a) Transition magnetic moment matrix elements phonon transition paths. a) Transition magnetic moment matrix elements (in
$\mu_{\mathrm{B}}$ ) for the connected states. b) Types of electron-phonon transitions: $\mu_{\mathrm{B}}$ ) for the connected states. b) Types of electron-phonon transitions:
multiphonon (solid green lines), strong one-phonon (bold dashed lines) and weak one-phonon (thin dashed lines). Red crosses denote quenched electronenergy phonons.

For the QTM relaxation process (3), it was taken into account that the only available electron-phonon escape transitions are to the states \pm 4 , with the rate $\sim \exp \left[-\left(E_{4}-E_{3}\right) / k T\right]$. Indeed, having in mind that $p_{-3} \exp \left[-\left(E_{-4}-E_{-3}\right)=p_{-4}\right)$, we obtain $\Gamma^{(4)} / \Gamma^{(3)}$ $=c_{4} / c_{3}$. However, $c_{4}$ and $c_{3}$ are just proportional to the squares of the transition matrix elements, with $\Gamma^{(4)} / \Gamma^{(3)} \approx 200$ at any temperature. Moreover, for incoherent tunnelling process, its rate, besides the square of the tunneling matrix elements, is proportional to the electron-phonon escape rate. The latter are direct processes from a given doublet to any other doublet (dashed vertical green arrows in Fig. 4b) having an exponential temperature dependence with an activation barrier equal to 
the energy of the corresponding transition. We therefore may conclude that all four relaxation processes have the temperature dependence $\exp \left(-E_{4} / k T\right)$, i.e. look like an activation processes with the blocking barrier $E_{4}$ (we have put $E_{1}=0$ ). This is in line with the experimental observation of one single blocking barrier with the height close to the calculated $E_{4}\left(1420 \mathrm{~cm}^{-1}\right.$ in Table S13, ESI). We want to stress that the high magnetization blocking barrier in $\mathbf{1}$ is not accompanied by the high axiality of several low-lying KDs as it was the case in all complexes with high blocking barrier investigated so far. This barrier arises solely from a lack of suitable high-energy phonons. Such a mechanism of building up of high blocking barrier was apparently never discussed in the past.

Despite high magnetization blocking barrier, the observed relaxation time in $\mathbf{1}$ is nonetheless short ( $<1 \mathrm{~s}$, Fig. 2c), which is due to appreciable non-axial anisotropy opening a tunneling relaxation path via interaction with the nuclear spins. Compared to the present complex, the best SMMs known to date differ only by smaller magnetic matrix elements and tunneling gaps (Fig. S14, ESI). These could be achieved in $\mathbf{1}$ as well if one manages to reduce somehow the equatorial crystal field. Thus, Fig. S18 (ESI) shows that the elongation of the four equatorial Dy-O bonds by 0.1 Á substantially reduces all magnetic matrix elements connecting states from the two sides of the barrier compared to the original geometry (Fig. 4a). Even a stronger effect could be achieved if weaker ligands such as nitrogen are used in the equatorial plane. The accompanying quench of the quantum tunneling of magnetization will allow a full exploitation of the high blocking barrier of the complex as in the best known SMMs.

\section{Conclusions}

In summary, we have described the synthesis and study of an octahedral dysprosium(III)-based complex with alkoxide $\mathrm{Ph}_{3} \mathrm{CO}^{-}$ligands located in the axial positions. The huge $\mathrm{CF}$ splitting induced by both, the short Dy-O axial distances and perfectly linear O-Dy-O angle leads to a genuine SMM behavior with a large anisotropic energy barrier of $1385 \mathrm{~cm}^{-1}$. Remarkably, such linear angle has never been achieved in the previously reported SMMs, while the energy barrier value appears very high for octahedral complexes. This fact is explained by an unprecedented mechanism of building up of the barrier consisting in the suppression of one-phonon transitions between the three low-lying CF multiplets due to large energy gaps between them exceeding the available phonon energies even in the absence of very high axiality of the excited doublets. Although it was never evidenced previously, this situation is not specific to the reported complex and could be generalized to other systems exhibiting giant CF splitting and lacking strong axiality. This is the major difference from the highly performant SMMs investigated up to now, where the high blocking barrier was achieved due to a strong axiality in a number of low-lying CF doublets.

\section{Acknowledgements}

The authors thank the University of Montpellier, CNRS and PAC of ICGM as well as KU Leuven. The Russian co-authors thank the Russian Science Foundation (grant 17-73-30036). The X-ray study has been carried out using an equipment of The Analytical Center of INEOS RAS. D. L. gratefully acknowledges Natural Science Basic Research Program of Shaanxi (program no.2020JQ-136) and the Fundamental Research Funds for the Central Universities"(G2020KY0519).").

\section{Notes and references}

1 S. Cotton, Lanthanide and actinide chemistry, John Wiley \& Sons, 2013.

2 J. Luzon and R. Sessoli, Lanthanides in molecular magnetism: so fascinating, so challenging, Dalton Trans., 2012, 41, 1355613567.

3 D. N. Woodruff, R. E. P. Winpenny and R. A. Layfield, Lanthanide single-molecule magnets, Chem. Rev., 2013, 113, 5110-5148.

4 F. Troiani and M. Affronte, Molecular spins for quantum information technologies, Chem. Soc. Rev., 2011, 40, 3119-3129.

5 L. Bogani and W. Wernsdorfer, Molecular spintronics using single-molecule magnets, Nat. Mater., 2008, 7, 179-186.

6 M. J. Giansiracusa, A. K. Kostopoulos, D. Collison, R. E. P. Winpenny and N. F. Chilton, Correlating blocking temperatures with relaxation mechanisms in monometallic single-molecule magnets with high energy barriers (Ueff > $600 \mathrm{~K}$ ), Chem. Commun., 2019, 55, 7025-7028.

7 L. Ungur and L. F. Chibotaru, Magnetic anisotropy in the excited states of low symmetry lanthanide complexes, Phys. Chem. Chem. Phys., 2011, 13, 20086-20090.

8 J. D. Rinehart and J. R. Long, Exploiting single-ion anisotropy in the design of f-element single-molecule magnets, Chem. Sci., 2011, 2, 2078-2085.

9 N. F. Chilton, Design Criteria for High-Temperature SingleMolecule Magnets, Inorg. Chem., 2015, 54, 2097-2099.

10 L. Ungur and L. F. Chibotaru, Strategies toward HighTemperature Lanthanide-Based Single-Molecule Magnets, Inorg. Chem., 2016, 55, 10043-10056.

11 J. Tang and P. Zhang, in Lanthanide Single Molecule Magnets, Springer Berlin Heidelberg, Berlin, Heidelberg, 2015, DOI: 10.1007/978-3-662-46999-6_2, pp. 41-90.

12 R. A. Layfield and M. Murugesu, Lanthanides and Actinides in Molecular Magnetism, Wiley, 2015.

13 A. Lunghi, F. Totti, R. Sessoli and S. Sanvito, The role of anharmonic phonons in under-barrier spin relaxation of single molecule magnets, Nat. Comm., 2017, 8, 14620.

14 C. A. P. Goodwin, F. Ortu, D. Reta, N. F. Chilton and D. P. Mills, Molecular magnetic hysteresis at 60 kelvin in dysprosocenium, Nature, 2017, 548, 439-442.

15 L. Escalera-Moreno, J. J. Baldoví, A. Gaita-Ariño and E. Coronado, Spin states, vibrations and spin relaxation in molecular nanomagnets and spin qubits: a critical perspective, Chem. Sci., 2018, 9, 3265-3275.

16 P. Evans, D. Reta, G. F. S. Whitehead, N. F. Chilton and D. P. Mills, Bis-Monophospholyl Dysprosium Cation Showing Magnetic Hysteresis at 48 K, J. Am. Chem. Soc., 2019, 141, 19935-19940. 
17 J. Liu, Y.-C. Chen, J.-L. Liu, V. Vieru, L. Ungur, J.-H. Jia, L. F. Chibotaru, Y. Lan, W. Wernsdorfer, S. Gao, X.-M. Chen and M.-L. Tong, A Stable Pentagonal Bipyramidal Dy(III) Single-lon Magnet with a Record Magnetization Reversal Barrier over $1000 \mathrm{~K}, \mathrm{~J}$. Am. Chem. Soc., 2016, 138, 5441-5450.

18 S. K. Gupta, T. Rajeshkumar, G. Rajaraman and R. Murugavel, An air-stable Dy(iii) single-ion magnet with high anisotropy barrier and blocking temperature, Chem. Sci., 2016, 7, 5181-5191.

19 Y.-S. Ding, N. F. Chilton, R. E. P. Winpenny and Y.-Z. Zheng, On Approaching the Limit of Molecular Magnetic Anisotropy: A Near-Perfect Pentagonal Bipyramidal Dysprosium(III) SingleMolecule Magnet, Angew. Chem. Int. Ed., 2016, 55, 1607116074.

20 F.-S. Guo, B. M. Day, Y.-C. Chen, M.-L. Tong, A. Mansikkamäki and R. A. Layfield, A Dysprosium Metallocene Single-Molecule Magnet Functioning at the Axial Limit, Angew. Chem. Int. Ed., 2017, 56, 11445-11449.

21 Y.-S. Meng, L. Xu, J. Xiong, Q. Yuan, T. Liu, B.-W. Wang and S. Gao, Low-Coordinate Single-lon Magnets by Intercalation of Lanthanides into a Phenol Matrix, Angew. Chem. Int. Ed., 2018, 57, 4673-4676.

22 A. B. Canaj, S. Dey, E. R. Martí, C. Wilson, G. Rajaraman and M. Murrie, Insight into D6h Symmetry: Targeting Strong Axiality in Stable Dysprosium(III) Hexagonal Bipyramidal Single-Ion Magnets, Angew. Chem. Int. Ed., 2019, 58, 14146-14151.

23 F.-S. Guo, B. M. Day, Y.-C. Chen, M.-L. Tong, A. Mansikkamäki and R. A. Layfield, Magnetic hysteresis up to 80 kelvin in a dysprosium metallocene single-molecule magnet, Science, 2018, 362, 1400-1403.

24 K. Randall McClain, C. A. Gould, K. Chakarawet, S. J. Teat, T. J. Groshens, J. R. Long and B. G. Harvey, High-temperature magnetic blocking and magneto-structural correlations in a series of dysprosium(iii) metallocenium single-molecule magnets, Chem. Sci., 2018, 9, 8492-8503.

25 Y.-S. Ding, K.-X. Yu, D. Reta, F. Ortu, R. E. P. Winpenny, Y.-Z. Zheng and N. F. Chilton, Field- and temperature-dependent quantum tunnelling of the magnetisation in a large barrier single-molecule magnet, Nat. Comm., 2018, 9, 3134.

26 Y. S. Ding, T. Han, Y. Q. Zhai, D. Reta, N. F. Chilton, R. E. P. Winpenny and Y. Z. Zheng, A Study of Magnetic Relaxation in Dysprosium(III) Single-Molecule Magnets, Chem. Eur. J., 2020, 26, 5893-5902.

27 K.-X. Yu, J. G. Kragskow, Y.-S. Ding, Y.-Q. Zhai, D. Reta, N. F. Chilton and Y.-Z. Zheng, Enhancing Magnetic Hysteresis in Single-Molecule Magnets by Ligand Functionalization, Chem, 2020, 6, 1777-1793.

28 H. Schumann and J. Müller, Metallorganische verbindungen der lanthaniden: VI. Neutrale und anionische alkyliden-komplexe von erbium und lutetium, J. Organomet. Chem., 1979, 169, C1C4.

29 S. J. Lyle and M. M. Rahman, Complexometric titration of yttrium and the lanthanons-I, Talanta, 1963, 10, 1177-1182.

30 F. Aquilante, J. Autschbach, R. K. Carlson, L. F. Chibotaru, M. G. Delcey, L. De Vico, I. Fdez. Galván, N. Ferré, L. M. Frutos, L. Gagliardi, M. Garavelli, A. Giussani, C. E. Hoyer, G. Li Manni, H. Lischka, D. Ma, P. Å. Malmqvist, T. Müller, A. Nenov, M. Olivucci, T. B. Pedersen, D. Peng, F. Plasser, B. Pritchard, M. Reiher, I. Rivalta, I. Schapiro, J. Segarra-Martí, M. Stenrup, D. G. Truhlar, L. Ungur, A. Valentini, S. Vancoillie, V. Veryazov, V. P. Vysotskiy, O. Weingart, F. Zapata and R. Lindh, Molcas 8: New capabilities for multiconfigurational quantum chemical calculations across the periodic table, J. Comput. Chem., 2016, 37, 506-541.

31 Y. C. Chen, J. L. Liu, Y. Lan, Z. Q. Zhong, A. Mansikkamaki, L. Ungur, Q. W. Li, J. H. Jia, L. F. Chibotaru, J. B. Han, W. Wernsdorfer, X. M. Chen and M. L. Tong, Dynamic Magnetic and Optical Insight into a High Performance Pentagonal Bipyramidal Dylll Single-Ion Magnet, Chem. Eur. J., 2017, 23, 5708-5715.

32 L. F. Chibotaru and L. Ungur, Ab initio calculation of anisotropic magnetic properties of complexes. I. Unique definition of pseudospin Hamiltonians and their derivation, J. Chem. Phys., 2012, 137, 064112.

33 D. Casanova, M. Llunell, P. Alemany and S. Alvarez, The Rich Stereochemistry of Eight-Vertex Polyhedra: A Continuous Shape Measures Study, Chem. Eur. J., 2005, 11, 1479-1494.

34 O. C. Gagné, Bond-length distributions for ions bonded to oxygen: results for the lanthanides and actinides and discussion of the f-block contraction, Acta Crystallographica Section B: Structural Science, Crystal Engineering and Materials, 2018, 74, 49-62.

35 J.-L. Liu, Y.-C. Chen and M.-L. Tong, Symmetry strategies for high performance lanthanide-based single-molecule magnets, Chem. Soc. Rev., 2018, 47, 2431-2453.

36 K. R. Meihaus, S. G. Minasian, W. W. Lukens, S. A. Kozimor, D. K. Shuh, T. Tyliszczak and J. R. Long, Influence of pyrazolate vs $\mathrm{N}$ heterocyclic carbene ligands on the slow magnetic relaxation of homoleptic trischelate lanthanide(III) and uranium(III) complexes, J. Am. Chem. Soc., 2014, 136, 6056-6068.

37 P. L. Scott and C. D. Jeffries, Spin-Lattice Relaxation in Some Rare-Earth Salts at Helium Temperatures; Observation of the Phonon Bottleneck, Phys. Rev., 1962, 127, 32-51.

38 S.-S. Liu, Y.-S. Meng, Y.-Q. Zhang, Z.-S. Meng, K. Lang, Z.-L. Zhu, C.-F. Shang, B.-W. Wang and S. Gao, A Six-Coordinate Dysprosium Single-lon Magnet with Trigonal-Prismatic Geometry, Inorg. Chem., 2017, 56, 7320-7323.

39 J.-L. Liu, K. Yuan, J.-D. Leng, L. Ungur, W. Wernsdorfer, F.-S. Guo, L. F. Chibotaru and M.-L. Tong, A Six-Coordinate Ytterbium Complex Exhibiting Easy-Plane Anisotropy and Field-Induced Single-lon Magnet Behavior, Inorg. Chem., 2012, 51, 8538-8544.

40 M. Guo and J. Tang, Six-Coordinate Ln(III) Complexes with Various Coordination Geometries Showing Distinct Magnetic Properties, Inorganics, 2018, 6, 16.

41 D. Gatteschi, R. Sessoli and J. Villain, Molecular nanomagnets, Oxford University Press on Demand, 2006.

42 Y.-C. Chen, J.-L. Liu, L. Ungur, J. Liu, Q.-W. Li, L.-F. Wang, Z.-P. Ni, L. F. Chibotaru, X.-M. Chen and M.-L. Tong, SymmetrySupported Magnetic Blocking at $20 \mathrm{~K}$ in Pentagonal Bipyramidal Dy(III) Single-Ion Magnets, J. Am. Chem. Soc., 2016, 138, 28292837.

43 M. Gregson, N. F. Chilton, A.-M. Ariciu, F. Tuna, I. F. Crowe, W. Lewis, A. J. Blake, D. Collison, E. J. L. McInnes, R. E. P. Winpenny and S. T. Liddle, A monometallic lanthanide bis(methanediide) single molecule magnet with a large energy barrier and complex spin relaxation behaviour, Chem. Sci., 2016, 7, 155-165.

44 F. Pointillart, B. le Guennic, O. Cador, O. Maury and L. Ouahab, Lanthanide Ion and Tetrathiafulvalene-Based Ligand as a "Magic" Couple toward Luminescence, Single Molecule Magnets, and Magnetostructural Correlations, Acc. Chem. Res., 2015, 48, 2834-2842.

45 J.-H. Jia, Q.-W. Li, Y.-C. Chen, J.-L. Liu and M.-L. Tong, Luminescent single-molecule magnets based on lanthanides: Design strategies, recent advances and magneto-luminescent studies, Coord. Chem. Rev., 2019, 378, 365-381. 
46 J. Long, Y. Guari, R. A. S. Ferreira, L. D. Carlos and J. Larionova, Recent advances in luminescent lanthanide based SingleMolecule Magnets, Coord. Chem. Rev., 2018, 363, 57-70.

47 J.-C. G. Bünzli and C. Piguet, Taking advantage of luminescent lanthanide ions, Chem. Soc. Rev., 2005, 34, 1048-1077.

48 L. Ungur and L. F. Chibotaru, Ab Initio Crystal Field for Lanthanides, Chem. Eur. J., 2017, 23, 3708-3718.

49 J. Long, I. V. Basalov, N. V. Forosenko, K. A. Lyssenko, E. Mamontova, A. V. Cherkasov, M. Damjanović, L. F. Chibotaru, Y. Guari, J. Larionova and A. A. Trifonov, Dysprosium SingleMolecule Magnets with Bulky Schiff-base Ligands: Modification of the Slow Relaxation of the Magnetization by Substituent Change, Chem. Eur. J., 2019, 25, 474-478.

50 D. A. Garanin and E. M. Chudnovsky, Thermally activated resonant magnetization tunneling in molecular magnets: $\mathrm{Mn}_{12}$ Ac and others, Phys. Rev. B, 1997, 56, 11102-11118.

51 A. Abragam and B. Bleaney, Electron paramagnetic resonance of transition ions, Oxford : Clarendon press, 1970. 